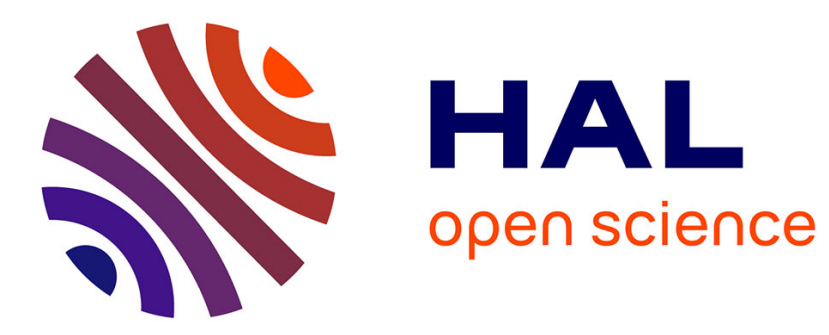

\title{
Self and collective correlation functions in a gel of tetrahedral patchy particles
}

Lorenzo Rovigatti, Francesco Sciortino

\section{To cite this version:}

Lorenzo Rovigatti, Francesco Sciortino. Self and collective correlation functions in a gel of tetrahedral patchy particles. Molecular Physics, 2011, 10.1080/00268976.2011.609148 . hal-00732531

\section{HAL Id: hal-00732531 \\ https://hal.science/hal-00732531}

Submitted on 15 Sep 2012

HAL is a multi-disciplinary open access archive for the deposit and dissemination of scientific research documents, whether they are published or not. The documents may come from teaching and research institutions in France or abroad, or from public or private research centers.
L'archive ouverte pluridisciplinaire HAL, est destinée au dépôt et à la diffusion de documents scientifiques de niveau recherche, publiés ou non, émanant des établissements d'enseignement et de recherche français ou étrangers, des laboratoires publics ou privés. 


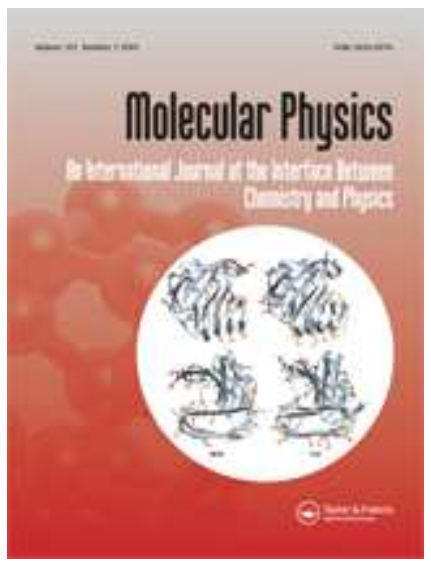

\section{Self and collective correlation functions in a gel of tetrahedral patchy particles}

\begin{tabular}{|c|c|}
\hline Journal: & Molecular Physics \\
\hline Manuscript ID: & TMPH-2011-0228 \\
\hline Manuscript Type: & Special Issue in honour of Luciano Reatto \\
\hline $\begin{array}{r}\text { Date Submitted by the } \\
\text { Author: }\end{array}$ & 05-Jul-2011 \\
\hline Complete List of Authors: & $\begin{array}{l}\text { Rovigatti, Lorenzo; Università di Roma La Sapienza, Physycs } \\
\text { Sciortino, Francesco; Università di Roma La Sapienza, Physics }\end{array}$ \\
\hline Keywords: & $\begin{array}{l}\text { tetrahedral network, glassy dynamics, patchy particles, equilibrium } \\
\text { gel }\end{array}$ \\
\hline \multicolumn{2}{|c|}{$\begin{array}{l}\text { Note: The following files were submitted by the author for peer review, but cannot be converted } \\
\text { to PDF. You must view these files (e.g. movies) online. }\end{array}$} \\
\hline rovigatti_sciortino.zip & \\
\hline
\end{tabular}

\section{SCHOLARONE ${ }^{\text {M }}$ \\ Manuscripts}


Molecular Physics

Vol. 00, No. 00, Month 200x, 1-10

\title{
RESEARCH ARTICLE
}

\section{Self and collective correlation functions in a gel of tetrahedral patchy particles}

\author{
Lorenzo Rovigatti ${ }^{a \dagger}$ and Francesco Sciortino ${ }^{b}$ \\ ${ }^{a}$ Dipartimento di Fisica, Università di Roma La Sapienza Piazzale A. Moro 5, 00185 \\ Roma, Italy \\ ${ }^{b}$ Dipartimento di Fisica and CNR-ISC, Università di Roma La Sapienza Piazzale A. \\ Moro 5, 00185 Roma, Italy \\ (Received 00 Month 200x; final version received 00 Month 200x)
}

\begin{abstract}
We discuss the results of intensive Brownian dynamics simulations of a simple model of tetrahedral patchy particles in the optimal network density region. This choice allows us to investigate the evolution of the structure and of the dynamics in a wide range of temperatures without encountering any phase separation. The slowing down of the dynamics in this model system is driven by the progressive bond formation and the increasing bond lifetime. Despite dynamical arrest is different from the glass case, where excluded volume interactions are dominant, the decay of the self and collective correlation functions of the resulting fluid bears similarities with the one observed in glassy systems.
\end{abstract}

\section{Introduction}

Patchy colloidal particles 1 3 continue to be the subject of an intense investigation, both experimentally and theoretically. There is indeed much expectation in the outcome of recent efforts of creating colloidal particles that interact via anisotropic potentials. The challenge faced by physicists, chemical engineers and material scientists is to organize these new geometries into structures for functional materials and devices via self-assembly, the spontaneous organization of matter into desired arrangements. The aim is to achieve - via the rational design of elementary building blocks (i.e. the particles) - pre-defined specific, ordered or disordered, structures, shifting from the top-down to the bottom-up approach, in which effort is made in the direction of controlling particle shape and patterning.

Theoretical and numerical studies of the phase behavior of patchy colloidal particles has been very much rewarding. Quite unexpectedly, a very rich framework for interpreting phenomena like thermoreversible gelation, the competition between gelation and glass transition or the competition between condensation and polymerization [4] has been unraveled. New concepts like empty liquids [5 7], equilibrium gels [8, 9], unconventional gas-liquid phase diagrams [10 12] have been introduced and have been very fruitful in promoting further developments [13, 14. One of the unexpected connections concerns the analogy between gelation in patchy colloids and glass formation in atomic and molecular network forming systems [13]. Indeed, studying the role of the "valence" $M$ (defined here as the maximum number of possible bonded nearest neighbors) it has been disclosed that the packing fraction $\phi$ of the liquid coexisting with the gas decreases on decreasing $M[5]$. For

${ }^{\dagger}$ Corresponding author. Email: lorenzo.rovigatti@uniroma1.it

ISSN: 0040-5167 print/ISSN 1754-2278 online

(C) 200x Taylor \& Francis

DOI: $10.1080 / 0040516$ YYxxxxxxxx

http://www.informaworld.com 
the case of hard-sphere colloids with four patches, $\phi$ is of the order of $30 \%$. This implies that, for larger values of $\phi$, gas-liquid phase separation is not encountered on cooling. On progressively decreasing temperature $T$, the average lifetime of a patch-patch bond increases and particles become arrested by being part of a longlived network of bonds. Energetic bonds thus determine the slowing down of the dynamics and the approach to a non-ergodic state, a dynamic arrest that we call gelation [6, 8, 15, 16]. Dynamic arrest can thus be expected to be different from the one characterizing glassy states, where caging is controlled by excluded volume interactions. Limited valence is crucial for observing gelation, since only when valence is limited this region of intermediate densities where packing does not play a major role is accessible at low $T$. For spherically interacting colloids, the gasliquid phase separation is much wider and the coexisting liquid density is found at $\phi \approx 0.6-0.7$. Hence, if crystallization is preempted, a liquid of spherically interacting particles can be brought to low $T$ to form a glass without phase separating only at very large densities.

In this article we investigate in details the evolution on cooling of the self and collective dynamics of a model for tetrahedral patchy colloids in the gel region at a fixed value of the density, in the so-called optimal network density region. In this window of densities the system is expected to be able to form an ideal (fully connected) random tetrahedral network[17]. At lower densities, gas-liquid phase separation takes place, while at larger densities packing prevents the possibility of geometrically arranging all molecules with proper angular and distance constraints required to form bonds. Despite the different nature of the dynamical arrest process, driven by bonding and not by packing, the decay of the correlation functions for this four coordinated model resembles the one observed in glasses.

\section{Models and numerical methods}

We study a simple continuous model for tetrahedral patchy particles by means of Brownian dynamics simulations. A particle is modeled as a rigid body defined by the position of its center of mass and by $M=4$ vectors indicating the locations of the four patches $[6$. The interaction potential between particles 1 and 2 is

$$
V(1,2)=V_{C M}+V_{P}
$$

where $V_{C M}$ is the potential acting between the centers of mass of the two particles, and $V_{P}$ is the interaction between patches:

$$
\begin{aligned}
V_{C M}(1,2) & =\left(\frac{1}{r_{12}}\right)^{m} \\
V_{P}(1,2) & =-\sum_{i=1}^{M} \sum_{j=1}^{M} \epsilon \exp \left[-\frac{1}{2}\left(\frac{r_{12}^{i j}}{\alpha}\right)^{n}\right] .
\end{aligned}
$$

The large value $m=200$ is chosen to approximate the hard-sphere behavior, the quantity $n=10$ makes the exponential function resemble a square well, $\alpha=0.12$ guaranties that the single bond per patch condition is satisfied and $\epsilon=1.001$ fixes the absolute minimum at unitary depth. The distance between the centers of particles 1 and 2 is indicated as $r_{12}$ while the distance between patches on different particles with the symbol $r_{12}^{i j}$. Bond forces thus act on surface spots allowing momenta which can induce particle rotations. 


\section{5,2011
Page 3

The parameters entering in the functional form (Eqs. 2 and 3) have been chosen in such a way that the resulting potential has a depth $u_{0}=-1$ and it resembles the one resulting from respectively an hard sphere and a square well potential, allowing greater flexibility in the study of the dynamics of these systems compared to stepwise potentials. Note that, while in the Kern-Frenkel potential[18 the interaction range and the angular width of the bond can be independently controlled, in the present continuous potential the patch-patch interaction depends only on the patchpatch distance and hence the patch-patch interaction range and angular width are coupled.

We perform Brownian dynamics simulations in the NVT ensemble using 10000 particles in a cubic box of size $L=26$ with periodic boundary conditions. In the following, the energy unit is chosen to be the depth $u_{0}$ of the potential, the length unit is chosen to be the colloids diameter $\sigma$ and time is in units of $\sigma \sqrt{m / u_{0}}$, where $m$ is the mass of the colloids. The Brownian algorithm used in the simulations is described in the appendix of Ref. [6]. Here we summarize its features. A Velocity Verlet integrator with a timestep $\delta t=0.001$ is used to integrate the equations of motion. To model Brownian diffusion, we define a probability $p$ for each particle to undergo a random collision every $N$ time steps. By tuning $p$ it is possible to obtain the desired free particle diffusion coefficient $D_{0}$ using the relation

$$
D_{0}=\frac{k_{B} T N \delta t}{m}\left(\frac{1}{p}-\frac{1}{2}\right)
$$

In the simulation units the chosen translational bare diffusion coefficient is $D_{0}^{T}=$ 0.01 and the corresponding rotational diffusion coefficient id $D_{0}^{R}=0.03$ (so that $D_{0}^{R} / D_{0}^{T}=3$, as expected for non-slip particles). These values fix $p^{T}$ and $p^{R}$ for each temperature.

The average time between two random collisions is given by

$$
\Delta t=\frac{N \delta t}{p}=\frac{k_{B} T N \delta t+2 m D_{0}}{2 k_{B} T}
$$

thus our simulations follow a Newtonian dynamics for $t<\Delta t$ and a Brownian dynamics after that time.

In order to equilibrate at the lowest temperatures we implemented a version of the code that runs on GPUs using CUDA[19]. The simulations were performed on Tesla C2050 GPUs. While on this hardware peak performances can be achieved only if single precision is used, the numerical instability makes it unfeasible to use float precision [20. To overcome these instabilities but still retain good performances we use double precision for time integration and single precision for force calculation [20, 21]. While using full double precision results in a two-fold performance loss, using this mixed single-double precision results only in a $10 \%-15 \%$ decrease in performances.

The performances achieved on GPUs depend heavily on interaction details (such as cut-off distances), number of particles and density 20 23]. For the state points investigated in this work $\left(N=10000, \rho \sigma^{3}=0.57\right)$ we obtained a $30 \mathrm{x}$ speed-up with respect to a Xeon E5620 (single core).

We have used up to $10^{10} \mathrm{MD}$ steps for equilibration and from $10^{7}$ to $2 \cdot 10^{9} \mathrm{MD}$ steps for data generation, depending on the temperature.

In Brownian dynamics simulations particles are subject to a random force which accounts for the collisions between colloidal particles and the solvent. Even if the random force has a zero mean, the position of the center of mass of the system becomes a random variable, again with zero mean. In standard simulations the 
random motion of the center of mass (COM) is negligible and the particle dynamics is weakly affected by this random process. In the study of very long simulations, as the ones reported here (which extend to $10^{9}$ integration time steps), the motion of the center of mass can be quite substantial and can produce artifacts in the evaluation of dynamical quantities. For this reason, in all data presented in this work the trajectories of the single particles have been corrected to subtract the center of mass motion. Care need to be taken in the analysis of Brownian dynamics trajectories in glassy states, especially now that the increased power of GPUs for scientific application is opening the possibility of investigating glassy states via lengthy simulations.

\section{Results}

\subsection{Static}

To proper frame the investigation of the dynamics, we start by showing in Fig. ??(a) the potential energy per particle as a function of $T$. The energy has the typical sigmoidal shape characteristic of bond interactions, reminiscent of the two-state behavior of the bonds (broken or formed). On cooling, the system changes from a collection of isolated clusters to a percolating network to an essentially fully bonded configuration, with a few isolated monomers, detaching from the infinite cluster, as indicated by the cluster size distribution, reported in Fig. ??(b). Two particles are considered connected (and hence belonging to the same cluster) if their pair interaction energy is lower than -0.5 . Below $T=0.15$, a large fraction of particles belongs to the infinite cluster and at the lowest investigated $T$, more than $99 \%$ of the particles is in the infinite cluster (see inset of Fig. ??(b)). Hence, at low $T$ the system can be visualized as a percolating network which incorporates most of the particles.

[Figure 1 here]

\subsection{Bond Lifetime}

The bond-bond autocorrelation function $C_{b}(t)$, defined as the probability that a bond existing at $t=0$ exists also at time $t$, provides a quantification of the typical microscopic time, setting the scale for the dynamics, separating the (short) time scale in which the dynamics takes place at fixed bonding pattern from the (long) time scale where dynamics is intrinsically connected to bond breaking events. Fig. ??(a) shows $C_{b}(t)$ for all the investigated $T$, showing that more than five order of magnitudes in bond lifetime are properly explored. The decay of the correlation function can be fitted with a stretched exponential function, $e^{-\left(t / \tau_{b}\right)^{\beta_{b}}}$, and the values of $\beta_{b}$ are reported in the inset, while the $T$ dependence of $\tau_{b}$ is shown in Fig ??(b). The decay is clearly stretched, suggesting that the different local bonding environments have a role in the process of bond breaking. From the fit, an average bond time can be calculated as $\left\langle\tau_{b}\right\rangle=\frac{\tau_{b}}{\beta_{b}} \Gamma\left(\frac{1}{\beta_{b}}\right)$, where $\Gamma(x)$ is the Gamma function.

[Figure 2 here]

\section{3. $\quad M S D$ and $D$}

Fig. ??(a) shows the mean square displacement (MSD) for all the investigated $T$. Above and around percolation, no plateau in the time dependence of the MSD is 


\section{uly 5,2011
Page 5 of

observed. Indeed, close to $T=0.15$, the lifetime of the bonds is still very short and no dynamical signatures of the presence of a transient infinite cluster are observed. Below percolation, an inflection develops which gives rise to a plateau which increases on further cooling. The height of the inflection point significantly changes with $T$, signaling that bonding progressively reduces the cage volume. At the lowest $T$, the MSD becomes comparable to the square of the bonding distance, suggesting that in a (almost) fully bonded locally tetrahedral structure, bond confinement can be quite effective.

The diffusion coefficient $D$, evaluated from the long time limit of the mean square displacement $(\mathrm{MSD}=6 D t)$ is shown in Fig. ??(b). $D$ is clearly super-Arrhenius around the percolation temperature. In this $T$-interval indeed the structure of the system changes significantly, since particles first aggregate into larger and larger clusters and, beyond percolation, join more and more the spanning cluster. As discussed later on, upon entering well inside percolation the structure of the system reaches its equilibrium gel state and no further significant structural changes take place. At these low $T, D$ shows an apparent Arrhenius behavior, with an activation energy of about -4.5 , a value slightly larger than the energy required to completely break four bonds. The Arrhenius dependence classifies the present model in the category of strong glass forming systems 24], which includes all tetrahedral network fluids. In this respect, the present results confirm once more that there is a strong connection between the insurgence of an open local structure held together by strong directional forces and the observation of an Arrhenius dynamics. It is also interesting to observe that a similar value has been recently reported in the study of ST2 water at the optimal network density[25]. Values of the activation energy of the order of four bonds have also been observed in a model of tetrahedral DNA constructs [26] and in a primitive model for water[17], suggesting that the mechanism for microscopic dynamics in tetrahedral networks shares common features.

[Figure 3 here]

\subsection{Self dynamics}

[Figure 4 here]

To analyze the tagged particle motion in the wave vector $\vec{q}$ space, we evaluate the self-intermediate scattering functions $F_{s}(q, t)$, defined as

$$
F_{s}(q, t)=\frac{1}{N} \sum_{i=1}^{N}<e^{-i \vec{q} \cdot\left(\vec{r}_{i}(t)-\vec{r}_{i}(0)\right)}>
$$

where $\vec{r}_{i}(t)$ is the position of the center of particle $i$ at time $t$. The behavior of the correlation functions is shown in Fig. ?? as a function of $T$ for two different wave-vector values ((a) and (b) panels) and as a function of $q$ at $T=0.10$ ((c) panel). To help comparing the characteristic timescales, the bond autocorrelation function is also reported. The long time decay of these functions can be rather well modeled via stretched exponentials,

$$
F_{s}(q, t)=f_{q}^{s} e^{-\left(t / \tau_{s}(q)\right)^{\beta_{q}^{s}}}
$$

where $f_{q}^{s}$ plays the role of the non-ergodicity factor, $\tau_{s}(q)$ is the characteristic decay time and $\beta_{q}^{s}$ is the stretching exponent. The wave-vector dependence of $f_{q}^{s}$, 


\subsection{Collective dynamics}

To analyze the collective particle motion in wave vector $\vec{q}$ space, we evaluate the coherent intermediate scattering functions $F_{c}(q, t)$, defined as

$$
F_{c}(q, t)=\frac{1}{N}<\sum_{i, j=1}^{N} e^{-i \vec{q} \cdot\left(\vec{r}_{i}(t)-\vec{r}_{j}(0)\right)}>
$$

As for the self-case, we show in Fig. ?? the $q$ and $T$ dependence of the collective correlation function. The data are significantly more noisy, reflecting the absence in the average over the distinct tagged particles. Despite the noise, some trends are clear and worth discussing. At low temperatures, very long times and small wave vectors, $F_{c}(q, t)$ shows an oscillatory behavior which has been tentatively attributed to the presence of acoustic sound modes $[8$. If we use Eq. 7 to fit these curves, we can note that also the collective non ergodicity parameter shows a clear $T$ dependence at low $T$. The values of $f_{q}^{c}$ indeed oscillate around the self ones, in phase with the position of the peaks of the structure factor $S(q)$ (Fig. ??(e)), similarly to what has been observed for glasses $[28-30$. Beside the main peak at $q \sigma \approx 8$, the structure factor shows a pre-peak characteristic of tetrahedral networks, where the slowest collective modes are found. On passing, we note that the $T$ dependence of $S(q)$ tends to saturate at low $T(S(q)$ at $T=0.10$ and $T=0.105$ are identical within the noise) suggesting that the system structure does not significantly evolve any longer with $T$. This saturation of $S(q)$ at small $T$ has been interpreted as evidence of an equilibrium gel state [5, 31.

Interestingly, the decay of the density fluctuations does not always requires the breaking of bonds. For example, at $T=0.115, \tau_{c}(q)$ is always smaller than $\tau_{b}$, suggesting that the decay of the density fluctuations at small $q$ happens at a fixed network structure. On further cooling, $\tau_{c}(q)$ and $\tau_{b}$ get closer, and at $T=0.10$ $\tau_{c}(q) \gtrsim \tau_{b}$ for intermediate and large values of $q$.

This means that the breathing modes of the network, diffusive in nature, are of sufficient amplitude to relax the density fluctuations at large wave-length. Only at very low $T$ the gel becomes so stiff that the decay of the density fluctuations takes 


\section{Page 7 of
Page

place on a time scale comparable or longer than $\tau_{b}$. This is more clearly shown in Fig. ??: at $T=0.115$ the bond-bond autocorrelation decays faster than the density fluctuations at $q \sigma=4.59$; at $T=0.105$ the two times are similar while at $T=0.10$ the opposite behavior is observed and the decay of the density fluctuations requires the breaking of the network to take place.

[Figure 6 here]

[Figure 7 here]

\section{Conclusions}

We have reported a study of the self and collective dynamics of a simple tetrahedral patchy model for a colloidal particle decorated by four attractive sites, located on the vertex of a tetrahedron. The shape and range of the site-site interaction is chosen in such a way that particles can form at most one bond per site. On cooling, the system progresses from a collection of isolated clusters to an essentially fully bonded tetrahedral network, in which most of the particles are engaged in four bonds. We have investigated the model at a fixed density, in the optimal network density region. Indeed, the presence of a limited number of strong directional interactions determines a limited range of densities which are compatible with the possibility of satisfying all possible interacting sites. This optimal density region is limited at low density by the presence of a gas-liquid coexistence and at high density by increasing packing, preventing the possibility of approaching the fully bonded network state. Along this isochore, the dynamics progressively slows down, first with a super-Arrhenious $T$ dependence (around percolation), then crossing to an Arrhenious dependence at low $T$. The behavior is similar to the one reported for silica and water, where also a cross-over from super-Arrhenious to Arrhenious has been observed in connection to the establishment of an extensively connected network 32, 33. In the case of silica this crossover has been interpreted as a manifestation of the Mode Coupling temperature 34. In this model, in which bonding is unambiguously defined, it appears that Arrhenious dynamics sets in when most of the particles belong to the spanning cluster. Interestingly, the comparison between the timescales of bond-breaking events and diffusional processes clearly shows that diffusion over long distances (as detected by $F_{s}(q, t)$ ) is slaved to the bond lifetime and a truly diffusional process (such that $\tau_{s} q^{2}$ is approximately constant) can be observed at very small wave vectors only. The characteristic timescale of the collective dynamics shows oscillations in phase with the structure factor, similarly to what has been found in the case of atomic and molecular glass formers 28 30]. Interestingly, the decay of the density fluctuations does not always requires the breaking of bonds. Only at very low $T$ the gel has become so stiff that the decay of the density fluctuations, even on length scales comparable to the particle size, requires the preliminary breakdown of the bond network. Under these conditions, the self and collective dynamic become slaved to the time scale set by $\tau_{b}$.

\section{Acknowledgments}

It is a great pleasure for us to contribute to the special issue in honor of Luciano Reatto, a leading scientist who has significantly contributed to shape, among others, the Italian liquid and soft matter communities. We acknowledge support from ERC-226207-PATCHYCOLLOIDS and ITN-234810-COMPLOIDS as well as from NVIDIA. 


\section{References}

[1]S.C. Glotzer and M.J. Solomon, Nat. Mat. 6, 557 (2007).

[2] A.B. Pawar and I. Kretzschmar, Macromol. Rapid Comm. 31, 150 (2010).

[3]E. Bianchi, R. Blaak and C.N. Likos, Phys. Chem. Chem. Phys. 13, 6397 (2011).

[4]F. Sciortino, E. Bianchi, J.F. Douglas and P. Tartaglia, J. Chem. Phys. 126, 4903 (2007).

[5]E. Bianchi, J. Largo, P. Tartaglia, E. Zaccarelli and F. Sciortino, Phys. Rev. Lett. 97, 168301 (2006).

[6] J. Russo, P. Tartaglia and F. Sciortino, J. Chem. Phys. 131, 014504 (2009).

[7]L. Rovigatti, W. Kob and F. Sciortino, ArXiv e-prints (2011).

[8]S. Saw, N.L. Ellegaard, W. Kob and S. Sastry, The Journal of Chemical Physics 134, 164506 (2011).

[9]R. Blaak, M.A. Miller and J.P. Hansen, Europhys. Lett. 78, 26002 (2007).

[10]J. Russo, J.M. Tavares, P.I.C. Teixeira, M.M. Telo da Gama and F. Sciortino, Phys. Rev. Lett. 106, 085703 (2011).

[11] A. Reinhardt, A.J. Williamson, J.P.K. Doye, J. Carrete, L.M. Varela and A.A. Louis, J. Chem. Phys. 134, 104905 (2011).

[12]F. Sciortino, A. Giacometti and G. Pastore, Phys. Rev. Lett. 103, 237801 (2009).

[13]F. Sciortino, Eur. Phys. J. B 64, 505 (2007).

[14]E. Zaccarelli, J. Phys.: Condens. Matter 19, 323101 (2007).

[15]E. Del Gado and W. Kob, Europhys. Lett. 72, 1032 (2005).

[16]E. Del Gado and W. Kob, Phys. Rev. Lett. 98, 028303 (2007).

[17]C. De Michele, S. Gabrielli, P. Tartaglia and F. Sciortino, J. Phys. Chem. B 110, 8064 (2006).

[18]N. Kern and D. Frenkel, J. Chem. Phys. 118, 9882 (2003).

[19]http://developer.nvidia.com/category/zone/cuda-zone

20] P.H. Colberg and F. Höfling, Computer Physics Communications 182, 1120 (2011).

[21] C.R. Trott, L. Winterfeld and P.S. Crozier, ArXiv e-prints (2010).

[22] J.A. Anderson, C.D. Lorenz and A. Travesset, J. Comput. Phys. 227, 5342 (2008).

[23]P.K. Jha, R. Sknepnek, G.I. Guerrero-García and M. Olvera de la Cruz, Journal of Chemical Theory and Computation 6, 3058 (2010).

[24]C.A. Angell, J. Non-Cryst. Solids 131-133, 13 (1991).

[25]P.H. Poole, S.R. Becker, F. Sciortino and F.W. Starr, ArXiv e-prints (2011).

[26]F.W. Starr and F. Sciortino, J. Phys.: Condens. Matter 18, L347 (2006).

[27]W. Götze, in Les Houches Summer Schools of Theoretical Physics Session LI (1989), edited by J.P. Hansen, D. Levesque and J.Zinn-Justin (North-Holland, Amsterdam, 1991), pp. 287-503.

[28]F. Sciortino, L. Fabbian, S.H. Chen and P. Tartaglia, Phys. Rev. E 56, 5397 (1997).

[29] A. Rinaldi, F. Sciortino and P. Tartaglia, Phys. Rev. E 63, 061210 (2001).

[30] J. Horbach and W. Kob, Phys. Rev. E 64, 041503 (2001).

[31]B. Ruzicka, E. Zaccarelli, L. Zulian, R. Angelini, M. Sztucki, A. Moussaïd, T. Narayanan and F. Sciortino, Nat. Mater. 10, 56 (2011).

[32] J. Horbach and W. Kob, Phys. Rev. B 60, 3169 (1999).

[33]F.W. Starr, F. Sciortino and H.E. Stanley, Phys. Rev. E 60, 6757 (1999).

[34]F. Sciortino and W. Kob, Phys. Rev. Lett. 86, 648 (2001). 
Figure captions:

(1) (a) Potential energy per particle $U$ as a function of temperature (black circles). Also shown for comparison is the potential energy per particle in a diamond crystal structure (red squares). (b) Number of clusters $n(s)$ of size $s$ for different temperatures for a system of 10000 monomers. For $T<0.15$ the system always contains a percolating cluster (disconnected points at $s \approx 10^{4}$ ). Inset: percentage of particles that are in the infinite cluster $\left(P_{\infty}\right)$ as a function of $T$.

(2) (a) Bond-bond autocorrelation function for different temperatures $(\mathrm{T}=0.098,0.10,0.105,0.11,0.115,0.12,0.15,0.25)$. A stretched exponential fit to $C_{b}(t)$ at $T=0.12$ is also included (dashed line). Inset: values of the fit parameter $\beta_{b}$ (see text) for different temperatures. (b) Values of the fit parameter $\tau_{b}$ (black circles) and the average bond time $\left\langle\tau_{b}\right\rangle$ (red squares, see text) for different temperatures.

(3) (a) Mean square displacement for different temperatures. The dashed line shows the expected time dependence of the diffusive behavior at long times for monomers (for which $D_{t}=0.01$ ). (b) Diffusion coefficient $D$ extracted from the slope of the MSD at long time for different temperatures (black circles). The red line is an Arrhenious fit performed over the five lowest temperatures. (4) (a) $F_{s}(q, t)$ at different temperatures for $q \sigma=0.48$. (b) $F_{s}(q, t)$ at different temperatures for $q \sigma=4.59$ (corresponding to the first peak in the $S(q)$, see Fig. ??(e)). (c) $F_{s}(q, t)$ at $T=0.10$ for different values of the wave vector $q \sigma$ $(0.24,2.1,3.9,5.7,7.5,9.3,11,13,15,17,18,20,22,24)$. Also shown is the $C_{b}(t)$ at the same temperature (dashed red line).

(5) Fit results for the self (solid lines) and collective (symbols) intermediate scattering functions for different temperatures as functions of the wave vector. (a) Non ergodicity factors. (b) Stretched exponents. (c) Characteristic decay times (dashed lines are $\tau_{b}$, added for comparison). (d) Characteristic decay times multiplied $q^{2}$. (e) Structure factors for the lowest temperatures.

(6) (a) $F_{c}(q, t)$ at different temperatures for $q \sigma=0.48$. (b) $F_{c}(q, t)$ at different temperatures for $q \sigma=4.59$. (c) $F_{c}(q, t)$ at $T=0.10$ for different values of the wave vector. Also shown is the $C_{b}(t)$ at the same temperature (dashed magenta line).

(7) $F_{c}(q, t)$ for $q \sigma=4.59$ (solid lines) and $C_{b}(t)$ (dashed lines) at three different temperatures. 